\section{Reformbedarf in der ambulanten, vertragsärztlichen Berufsausübung - Forderungen der Radiologie an die neue Bundesregierung (2. Teil)}

Der 1. Teil dieses Beitrages beschäftigte sich insbesondere mit der Benachteiligung ärztlicher Berufsausübungsgemeinschaften gegenüber MVZ und der Tatsache, dass angestellte Ärzte im Vertragsarztrecht keinerlei Rechts- und Teilnahmestatus besitzen. In dem hier folgenden 2. Teil werden insbesondere Fragen der Bildung von BAG zwischen MVZ Trägergesellschaften, die Einschränkung der Berufsausübung durch bedarfsplanungsrechtliche Vorgaben und das Verhältnis des Nachbesetzungsverfahrens zu Verzicht und Anstellung behandelt.

\section{BAG zwischen MVZ Trägergesellschaften}

Wie bereits oben ausgeführt, können sich MVZ untereinander oder mit Vertragsärzten zu einer (überörtlichen) BAG nach $\S \S 1 \mathrm{a}$ Nr. 12, 15c BMV-Ä zusammenschließen.

In einer BAG wird die vertragsärztliche Tätigkeit von zur vertragsärztlichen Versorgung zugelassenen Leistungserbringern ganz oder teilweise gemeinsam ausgeübt; es handelt sich um eine nach außen in Erscheinung tretende, auf Dauer angelegte, Behandlungsgemeinschaft mit gemeinsamem Patientenstamm, welche der vorherigen Genehmigung seitens des Zulassungsausschusses bedarf ( $§ 33$ Abs. 3 S. 1 ÄrzteZV). Mit der Genehmigung wird den Mitgliedern der BAG eine besondere Form der Berufsausübung gestattet ${ }^{1}$. Diese ist nach Ansicht des BSG durch eine gemeinsame Ausübung der ärztlichen Tätigkeit durch mehrere Ärzte der gleichen oder ähnlicher Fachrichtung in gemeinsamen Räumen mit gemeinsamer Praxiseinrichtung, gemeinsamer Karteiführung und Abrechnung sowie mit gemeinsamen Personal auf gemeinsame Rechnung geprägt ${ }^{2}$.

1 BSG, Urt. v. 15.8.2012, Az.: B 6 KA 47/11 R, NZS 2013, 155 (156).

2 BSG, Urt. v. 14.12.2011, Az.: B 6 KA 31/10 R, MedR 2012, 826 (827).
Schließen sich hingegen z. B. zwei MVZ Trägergesellschaften in der Rechtsform der $\mathrm{GmbH}$ zu einer BAG zusammen, handelt es sich um einen Zusammenschluss von juristischen Personen mit angestellten Ärzten. Die ärztliche Berufsausübung findet daher in den MVZ und nicht in der BAG statt. Da diese Form der BAG jedoch im BMV-Ä als zulässig angesehen wird, bedarf es für diese Form der BAG ebenfalls entsprechende konkretisierende untergesetzliche Regelungen. Bisher existieren hierzu in der Ärzte-ZV und dem BMV-Ä, anders als für freiberuflich tätige Ärzte in $§ 32$ Abs. 1 Ärzte-ZV, keine Vorschriften, die diese Form der „Berufsausübung“ normieren.

\section{„Gründe der vertragsärzt- lichen Versorgung" als Berufsausübungshindernis}

Mit dem Vertragsarztrechtsänderungsgesetz (VÄndG) 3 hat der Gesetzgeber eine deutliche Flexibilisierung und Liberalisierung der ärztlichen Berufsausübung in der ambulanten vertragsärztlichen Versorgung angestrebt. Ausweislich der Gesetzesbegründung zum VÄndG sind die Änderungen im Vertragsarztrecht als Folge des 104. Deutschen Ärztetages in Bremen eingeführt worden, um die „durch die neuen (Muster-)Berufsordnungen geschaffenen Spielräume für die Berufsausübung der niedergelassenen Ärzte, Zahnärzte und Psychotherapeuten im vertragsärztlichen, vertragszahnärztlichen und vertragspsychotherapeutischen Alltag wirksam werden zu lassen“. Darüber hinaus hat es der Gesetzgeber zur Herstellung effizienter und auch medizinisch sinnvoller Versorgungsstrukturen in einigen Bereichen sogar als notwendig angesehen, „im Vertragsarztrecht über die im ärztlichen Berufsrecht erfolgte Liberalisierung hinauszugehen“4.

3 VÄndG v. 22.12.2006, BGBI. I, S. 3439.

4 Vgl. BT-Drs. 16/2474, S. 15, 16.

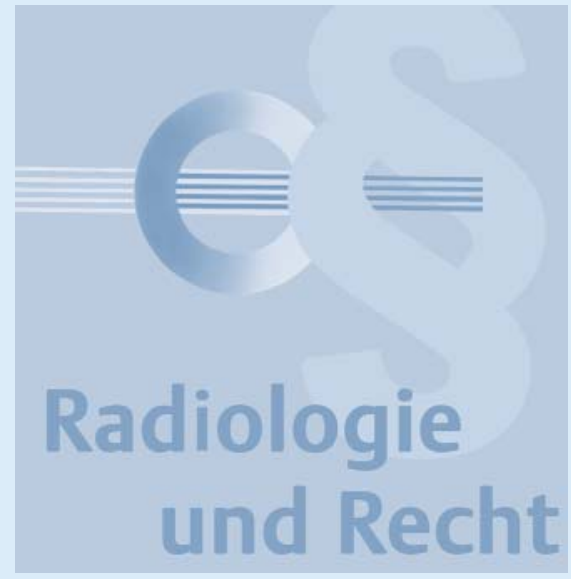

In der Folgezeit ist jedoch eine Entwicklung zu beobachten, die sukzessiv die geschaffenen Möglichkeiten und Freiheiten der ärztlichen Berufsausübung wieder einschränkt oder sie gar zurücknimmt. Seit dem VÄndG sind in den nachfolgenden Gesundheitsreformen zunehmend Bedarfsplanungsgesichtspunkte in der Gestalt unbestimmter Rechtsbegriffe in das Gesetz aufgenommen worden, die die ursprünglichen Liberalisierungstendenzen in der vertragsärztlichen Berufsausübung zunehmend in das Gegenteil verkehren. Die durch das GKV-VStG vorgenommene Reform der vertragsärztlichen Bedarfsplanung wurde sukzessive durch ein System der einzelfallbezogenen Bedarfsprüfung ergänzt, welches auf unbestimmten Rechtsbegriffen beruht und mangels weitergehender gesetzlicher Ausgestaltung den KVen und Zulassungsausschüssen zur Ausfüllung im Einzelfall überlassen bleibt. Dies hat dazu geführt, dass in den Fällen, in denen eine zulassungsrechtliche Entscheidung von einer Bedarfsprüfung abhängig ist, mangels entsprechender Kriterien bundesweit unterschiedliche Auslegungen der Bestimmungen und Entscheidungen der Zulassungsgremien gegeben sind und Anträge von Vertragsärzten z. T. mit willkürlichen Argumenten abgelehnt werden.

Exemplarisch lässt sich dies an dem negativen Tatbestandsmerkmal „kein Entgegenstehen von Gründen der vertragsärztlichen Versorgung“ belegen. Obgleich sich dieses Kriterium an mehreren Stellen im Gesetz wiederfindet (Nachbesetzungsverfahren, $\S 103$ Abs. 3a, 4 SGB V; Verzicht und Anstellung, § 103 Abs. 4a und 4b SGB V; Verlegung von Vertragsarztsitzen und Anstellungen §24 Abs. 7 Ärzte-ZV; Filialgenehmigung, 
§24 Abs. 3 Ärzte-ZV) und ihm eine erhebliche Bedeutung zuteilwird, sind dem Gesetz weder eine Legaldefinition noch entsprechende Ausführungsbestimmungen zu entnehmen. Es kommt hinzu, dass die Zulassungsgremien nach der Rechtsprechung einen sehr weiten Beurteilungsspielraum bei der Auslegung von Bedarfsplanungsgesichtspunkten und damit dieser unbestimmten Rechtsbegriffe haben. Zugleich ist dieser Beurteilungsspielraum auf Grund des Gewaltenteilungsgrundsatzes (Exekutive einerseits und Judikative andererseits) gerichtlich nur eingeschränkt überprüfbar ${ }^{5}$, da es sich bei ihnen um sog. pluralistisch besetzte Wertungsgremien handelt, welche eine unabhängige und sachverständige Entscheidung treffen.

Die gerichtliche Kontrolle beschränkt sich darauf, ob der Verwaltungsentscheidung ein richtig und vollständig ermittelter Sachverhalt zugrunde liegt, ob die Grenzen der Auslegung eingehalten und ob die Subsumtionserwägungen so hinreichend in der Begründung der Entscheidung verdeutlicht wurden, dass im Rahmen des Möglichen die zutreffende Anwendung der Beurteilungsmaßstäbe erkennbar und nachvollziehbar ist. Die seitens des Zulassungsausschusses angestellten Wertungen und Beurteilungen sind daher der Prüfungskompetenz eines Gerichtes entzogen und können damit auch gerichtlich nicht angegangen werden, sofern keine sachfremden Erwägungen angestellt und die zwingenden gesetzlichen Vorgaben beachtet wurden. Dies führt dazu, dass die Auslegung des Gesetzes in den KVen unterschiedlich gehandhabt wird. Dies hat zur Konsequenz, dass zwar die einzelne Entscheidung der jeweiligen KV zwar frei von Willkür sein muss (Verbot sachfremder Erwägungen). Es schützt aber nicht davor, dass die KVen ein und denselben Sachverhalt einheitlich beurteilen müssen, infolgedessen ein Vergleich sämtlicher Entscheidungen dazu führen kann, dass sie insgesamt willkürlich sind. Letztlich entscheidet die jeweilige Zugehörigkeit zu einer KV darüber, ob der Antrag des Leistungserbringers in seinem Sinne beschieden wird.

5 Etwa BSG, Urt. v. 3.8.2016, Az.: B 6 KA 31/15, NZS 2017, 109 (112).
Diese Bedarfsprüfung führt im Bereich des Nachbesetzungsverfahrens nach $\S 103$ Abs. 3a, 4 SGB V, auch nach Auffassung der meisten Zulassungsausschüsse, dazu, dass eine praktische und rechtssichere Handhabung dieses Instrumentariums nicht mehr möglich ist. Wir befinden in einem Rechtsbereich der durch Detailregelungen und Überregulierung nicht mehr handhabbar und daher nicht mehr umsetzbar ist.

\section{Verzicht und Anstellung nach $\S 103$ Abs. 4a und 4b SGB V}

Demgegenüber kann außerhalb des Nachbesetzungsverfahrens und insbesondere ohne Auswahlverfahren und Begrenzung der Praxiskaufpreise durch eine Verkehrswertermittlung weiterhin eine freie Übertragung vertragsärztlicher Zulassungen auf MVZ und BAGs im Rahmen der Verzichtsregelungen in $\S 103$ Abs. 4a und 4b SGB V erfolgen. Es ist kein Wunder, dass dieses zulassungsrechtliche Instrument in der Praxis zum Regelfall der Übertragung vertragsärztlicher Zulassungen geworden ist und das Nachbesetzungsverfahren nur zum Tragen kommt, wenn das Ergebnis der Nachbesetzung aufgrund entsprechender Privilegierungstatbestände (z. B. Nachbesetzung in einer BAG) bereits vorher rechtssicher feststeht oder keine andere Übertragungsmöglichkeit besteht.

Das BSG ${ }^{6}$ hat zwar die Möglichkeiten zur Umgehung des Nachbesetzungsverfahrens dadurch etwas erschwert, in dem es festgestellt hat, dass das Recht zur Nachbesetzung einer in ein MVZ im Wege des Verzichts nach $\S 103$ Abs. 4a SGB V eingebrachten Stelle einem MVZ grundsätzlich nur zusteht, wenn der Arzt dort mindestens drei Jahre tätig war. Zu Recht führt das BSG aus, dass für diesen Fall „weder eine Entscheidung des Zulassungsausschusses zum ,ob' der Nachbesetzung noch eine Auswahlentscheidung zwischen mehreren Bewerbern um die Praxisnachfolge vorgesehen “ ist, wie dies das Nachbesetzungsverfahren nach §103 Abs. 3a, 4 SGB V

6 BSG, Urt.v. 4.5.2016, Az.: B 6 KA 21/15 R, MedR 2016, 1006 (1010). erfordert. Vielmehr ist dem MVZ die Genehmigung zur Anstellung des Arztes zu erteilen, der auf seine Zulassung verzichtet hat. Der Grund dafür, warum die Anstellungsgenehmigung beim Vorliegen der gesetzlichen Voraussetzungen zu erteilen sei, ohne dass dem Zulassungsausschuss ein Entscheidungsspielraum verbleibe, liegt nach Ansicht des BSG darin, dass der Vertragsarzt bei dieser Variante des Zulassungsverzichts seine Tätigkeit innerhalb des Systems der vertragsärztlichen Versorgung und nur mit einem anderen Status dem des Angestellten - fortführe. Demgegenüber sei die Nachbesetzung auf ein Ausscheiden aus dem System angelegt.

Auch wenn durch die Anforderung einer mindestens dreijährigen Tätigkeit in dem MVZ nach § 103 Abs. 4a SGB V oder bei einem Vertragsarzt nach $\S 103$ Abs. 4b SGB $\checkmark$ die nicht akzeptable Umgehung des Nachbesetzungsverfahrens zumindest deutlich eingeschränkt wird, stellt sich die Frage, ob es hingenommen werden kann, dass der Gesetzgeber mit dem GKV-VStG einerseits Regelungen aufgenommen hat, nach denen die Zulassungsausschüsse auf die Nachbesetzung von Arztsitzen in überversorgten Planungsbereichen ganz verzichten müssen, andererseits aber mit dem Verzichtsverfahren weiterhin eine unbeschränkte Übertragungsmöglichkeit von Zulassungen besteht.

Zu berücksichtigen ist, dass die Verzichtsregelung zugunsten eines MVZ in $\S 103$ Abs. 4a SGB V durch das GKV-Modernisierungsgesetz $(G M G)^{7}$ zur Stärkung der mit diesem Gesetz neu eingeführten MVZ aufgenommen worden ist. In Verbindung mit der Erteilung der bedarfsunabhängigen Zulassung nach einer fünfjährigen Tätigkeit in einem MVZ sollte die Attraktivität der MVZ für junge Ärzte erhöht werden:

„Durch diese Möglichkeiten der „Übertragung “ der Zulassungen in ein medizinisches Versorgungszentrum werden die Möglichkeiten der Neugründung von Zentren verbessert, da auch bei Sperrung wegen Überversorgung neue Zentren gegründet werden können. Da die Übertragung „bedarfsplanungsneutral" erfolgt, wird gleichzeitig vermieden, dass

7 GMG v. 14.11.2003, BGBI. I, S. 2190. 
es zur Steigerung der Zahl der vertragsärztlichen Leistungserbringer kommt. “8

Dieses gesundheitspolitische Bedürfnis der Stärkung von MVZ ist heute nicht mehr gegeben. Demgegenüber stellt sich die Frage, ob die Konkurrenz zwischen Nachbesetzungsverfahren und Verzichtsregelung der Forderung nach einem geordneten Auswahlverfahren für eine exklusiv zu vergebende Position gerecht wird. Das BSG hat im Zusammenhang mit der Entscheidung über die Unzulässigkeit des sog. „Windhundprinzips “ in seiner Entscheidung vom 19.10.2011 ${ }^{9} \mathrm{zu}$ Recht darauf hingewiesen, dass der Ausgestaltung des Auswahlverfahrens nach $\S 103$ Abs. 4 SGB V im Lichte des Grundrechts aus Art. 12 Abs. 1 GG erhebliche verfahrensrechtliche Bedeutung zukomme. Dieses diene insbesondere dem durch Art. 12 Abs. 1i. V.m. Art 3 Abs. 1 GG gewährleisteten Anspruch auf Chancengleichheit. Diesem Anspruch wird jedoch die gegenwärtige Praxis des Zulassungsverzichts, die jenseits des stark reglementierten Nachbesetzungsverfahrens eine relativ unbeschränkte Übertragungsmöglichkeit der Zulassung und des Praxisverkaufs ermöglicht, nicht mehr gerecht.

Die Instrumente des Zulassungsverzichts zugunsten einer Anstellung sollten daher daraufhin überprüft werden, ob diese in der jetzigen Form mit den Anforderungen an eine Chancengleichheit der Bewerber noch gerecht werden und ob es hingenommen werden kann, dass dem Auswahlverfahren im Nachbesetzungsverfahren zahlreiche Zulassungen entzogen werden.

\section{Ergebnis}

Die ambulante vertragsärztliche Versorgung befindet sich in einem grundlegenden Strukturwandel. Zum einen müssen insbesondere geräteintensive Facharztgruppen immer größere Praxisstrukturen aufbauen, um ihre Investitionen refinanzieren zu können und wettbewerbsfähig zu bleiben. Dies ist jedoch nur im Rahmen liberaler vertragsarztrechtlicher Strukturen möglich. Sowohl der Gesetzgeber, als auch die Judikatur ebenso wie vereinzelte KVen wirken diesem Bedürfnis jedoch entgegen. Zum anderen ist seit einiger Zeit eine zunehmende Konkurrenz zu den traditionell geführten ärztlichen BAG durch solche MVZ festzustellen, die durch Krankenhäuser und andere nichtärztliche Leistungserbringer gegründet werden. Verdrängungsaktivitäten kapitalstarker kommerzieller Anbieter können die wirtschaftliche Leistungserbringung bestehender Praxen jedoch auf absehbare Zeit unmöglich machen. ${ }^{10}$ Dadurch wird es für niederlassungswillige Ärzte und bestehende BAGs zunehmend schwieriger, Praxen zu gründen oder zu erweitern. Die Folge ist eine fortschreitende Zentralisierung der Versorgung.

Um dieser Entwicklung entgegenzutreten, ist es einerseits erforderlich, dass die rechtliche und verfassungsrechtlich nicht zu rechtfertigende Benachteiligung der niedergelassenen Vertragsärzte gegenüber MVZ hinsichtlich der Gestaltungsmöglichkeiten beendet wird. Über diese Gleichstellung hinaus bedarf es andererseits einer Stärkung der BAG nach §33 Abs. 2 Ärzte-
ZV. Des Weiteren sollte die aus dem Gleichgewicht geratene Dichotomie der Gestaltungsmodelle Nachbesetzungsverfahren einerseits und Anstellung und Verzicht andererseits wiederhergestellt wird. Zusätzlich müssen die aufgezeigten Detailfragen gesetzlich geklärt werden, damit eine einheitliche und vor allem rechtssichere Rechtsanwendung und Gestaltung ermöglicht wird. Die momentan zu beobachtende Schwächung der niedergelassenen Vertragsärzte stellt die durch sie garantierte Sicherstellung in dem notwendigen Umfang auf hohem qualitativen Niveau in Frage. Das Credo muss daher insgesamt lauten, dass die Freiberuflichkeit niedergelassener Vertragsärzte gestärkt werden muss, damit die ärztliche Berufsausübung im Rahmen von BAG und MVZ auch zukünftig attraktiv und lohnenswert ist. Nicht zuletzt bedarf es einer Stärkung der Rechte des angestellten Arztes, indem ihm beispielsweise partielle (Antrags-) Rechte im Zulassungsverfahren eingeräumt werden.

Prof. Dr. Peter Wigge

Rechtsanwalt

Fachanwalt für Medizinrecht

Rechtsanwälte Wigge

Scharnhorststraße 40

48151 Münster

Telefon: (0251) $53595-0$

Telefax: (0251) 53595 - 99

E-Mail: kanzlei@ra-wigge.de www.ra-wigge.de
8 BT-Drs. 15/1525, S. 112.

9 BSG, Urt. v. 19.10.2011, Az.: B 6 KA 20/11 R, MedR 2012, 540 (542f.).
10 So schon Köhler, Gegen den industriellen Verdrängungswettbewerb, DÄBI. 2002, A- 828 (829). 